\title{
An introductory concept of evidence for medicine
}

\author{
Patrick S. Phelan \\ Division of Public Health Sciences, Department of Surgery \\ Washington University School of Medicine in St. Louis
}

October 24, 2018

Word Count: 2307 (footnotes: 103) 
For new medical students, the word "evidence" is introduced early and repeated often in the context of "evidence-based medicine," (EBM) a framework to which substantial portions of modern curricula are devoted. This starting point seems fitting for the present discussion, as the EBM name itself has been controversial; failure to formally delimit the term may have underlain initial confusion regarding the domain of EBM, as even seminal attempts at clarification lack explicit definition of what it is to be "evidence."-2 This essay has been motivated, in part, by the observation that this question of "evidence" is not always posed to students, though it forms an implicit foundation for both clinical and scientific reasoning. Whereas theoretical treatment of this topic is extensive and evolving, I suggest that a simplified definition, proposed herein, ought to be considered as an introduction to critical evaluation of what is, and what it is to be evidence.

\section{Evidence in General}

Appropriately, a recent exploration of the conceptual nature of evidence comes from within the field of medical education: Thistlethwaite and colleagues provide a record of topical discussion at the 2011 Association for Medical Education in Europe symposium, where multidisciplinary, qualitative perspectives were considered on the view of evidence. ${ }^{3}$ The discussants found evidence to be a complex construct intimately linked with its use in rhetoric, and simultaneously embedded in and molded by social and scientific context. Rather than offering a summative definition of the term, the authors view its meaning as fluid and pluralistic, to be interpreted in light of its intended purpose, its source, and even the identity of its interpreters. 
From this view, it should be unsurprising that questions of evidence cover well-trodden ground in the realm of philosophy. In an overview of relevant theory, Kelly explores the major conceptions of evidence and its functions ${ }^{4}$ As a point of distinction, he first notes that common lay and legal concepts of evidence consider the substance of propositional support, ${ }^{4}$ (e.g. "physical evidence") a unity reflected in many English dictionary definitions and in contrast to more abstract considerations of the nature of evidence. In this latter sense, important themes include the role of evidence in justifying belief, its centrality to rationality, and its relationship with objectivity. ${ }^{4}$ Setting aside nuanced epistemological questions of what evidence is, considerations under these umbrellas highlight the apparent subjectivity of evidence in multiple respects.

Foremost, merely recognizing evidence as such may be dependent on background knowledge, that is, on one's theoretical foundation. Appraisal of evidence then arguably requires its consideration with respect to some proposition of interest, for which it may be highly relevant what competing propositions vie for support. Whereas theoretical foundations and known propositions often differ between observers, it is presumably in this arena that evidence arbitrates in a so-called "intersubjective" manner. ${ }^{4}$ However, though evidence appears to play a natural role in supporting objective inquiry, its use seems reciprocally to presuppose some degree of objective appraisal. Perspectives on these issues span the fields of epistemology and philosophy of science. Nevertheless, the general impression is that evidence (whatever it is) ought to relate reason with belief in a way somewhat amenable to objective evaluation. One approach operationalizes evidence in terms of a "probability calculus" whereby observations 
provide evidence inasmuch as they change existing probabilities of the truth of hypotheses, and is closely related to Bayesian inference ${ }^{4}$ (the same framework adopted for clinical reasoning in EBM).

Taking a statistical-philosophical approach in this vein, Morey and colleagues consider quantitative evidence under the Bayesian paradigm. ${ }^{5}$ They propose that "the evidence presented by data is given by the impact that the data have on our evaluation of a theory." They consider as key characteristics that this impact is both relational, that is, "fundamentally about the relation between data and hypotheses, and not data alone," and relative, (i.e. comparative) that is, such that "evidence for or against a hypothesis can only be assessed relative to another hypothesis." Like Davies, ${ }^{2}$ the authors see data as "inanimate, ${ }^{3}$ "dry,"5 and uninformative absent any theoretical context or interpretation.

\section{Evidence in Medicine}

What are the implications of these theoretical considerations on the meaning of "evidence" within medicine? This question has been explored by several authors over the past two decades.

In a particularly relatable example, Macnaughton (1998) depicts evidence through the paired lenses of the scientist and the detective, identifying core characteristics in each case to inform a concept of medical evidence. ${ }^{6}$ In both contexts, information is said to become evidence only if it bears relevance to evaluation of a hypothesis, but the scientist's evidence is distinguished from that of the detective by its generality: whereas the scientist seeks to know universal truths of 
nature, the detective desires the truth of a single case, and other cases may differ. For the physician, identification and use of clinical evidence appears to require a hybrid perspective, and Macnaughton emphasizes the role of the patient as both a source of case-specific evidence and the context for application of scientific theory. ${ }^{6}$

The generality distinction above is echoed by Fox (2011) in comparing legal and scientific evidence, adding that in the former case the broad inclusivity of "evidence" has invited the suggestion that the term be reserved for the "process by which truth is established, and the term proof [for] the effect which the evidence produces upon the judicial mind" (first emphasis added). ${ }^{7}$ Fox also notes that a "traditional" definition of "evidence" holds that it constitutes "information" that alters confidence in uncertain propositions. ${ }^{i}$ Although Fox accepts a provisional construction of evidence as "any information that is relevant to making a decision about a hypothesis," (original emphasis) this appears to be an outlier among philosophically sophisticated discussions on the subject. ${ }^{7, i i}$

\section{Toward an Introductory Concept}

Upon inspection, the traits of relationality and relativity are apparent (though not mandated) throughout the preceding explorations, and it seems natural that an introductory answer to the question of "evidence" should call upon these characteristics. More comprehensively, Upshur (2000) identifies "seven characteristics of medical evidence" most informative to its epistemological situation, arguing that medical evidence should be considered through the lens

of fallibilism. ${ }^{8}$ In such a perspective the quality of defeasibility is key, but of greater interest to 
the present topic is Upshur's characteristic of emergence, for which it can be inferred from the given examples that the apparent strength and utility of evidence are colored by the broader context in which it arises.

Subsequently, Upshur and colleagues (2001) developed a "taxonomy of evidence" modeled as a Cartesian plane with "meaning" versus "measurement" orienting one axis, and "particular" versus "general" contexts orienting the other. ${ }^{9}$ The authors' implication is that evidence can be understood not merely to refer to some data, but "as a mediation between the context of its use and the method of its production."

Across these varied perspectives a common theme is clear: evidence is something greater than mere information. A concise yet broadly applicable introductory concept of evidence then ought to reflect this unambiguously. Thus, I propose we take "evidence" to be that property of data which inform on the truth of hypotheses. Hypotheses is intended as broadly inclusive of propositions, from clinical diagnoses to research predictions, whether qualitative or formalized within statistical models. Similarly, data need not be quantitative nor derived under research conditions, but may be mere observations (e.g. upon a portion of the physical exam). Furthermore, this definition does not intend evidence as a uniform trait, but something of a vector denoting the degree of support provided and the hypothesis (or hypotheses) to which support is directed. 
In arguing for the superiority of this evidence-as-property approach, I am departing somewhat from the definitions (admittedly, often simplified) given by most authors, but this distinction is not entirely novel. Indeed, Goodman and Royall (1988) invoke it explicitly:

Evidence is a property of data that makes us alter our beliefs...[it] is the basis upon which we derive inferences. ${ }^{10}$ (p. 1568)

Though the authors do not explore the epistemological nature of this property further, they do situate it within the mathematical likelihood paradigm in which both relativity and relationality are key:

...the measurement of evidence requires at least three components: the observations, and two competing explanations for how they were produced. ${ }^{10}$ (p. 1569)

Goodman and Royall's mathematical formalization of evidence is highly instructive, and like that of Morey and colleagues, fits neatly with the Bayesian construction of quantitative clinical reasoning in EBM. ${ }^{\mathrm{iii}}$ However, I argue it is not essential for a meaningful introduction to the concept of evidence. As Upshur and colleagues (2001) demonstrate, the realm of evidence spans the quantitative and qualitative, ${ }^{9}$ and I argue that thinkers new to theory of evidence will be best served by a most general statement of its nature.

\section{Implications of Evidence-as-Property}

How we conceive of evidence has important implications for how we use our language. On one view, the word "evidence" is simply a collection of glyphs serving as a placeholder for the more complex concept that is the subject of this essay. To the extent that the concept of evidence is understood to concern relations of ideas, the mere label "evidence" may be considered 
secondary. On a broader view however, if the concept of evidence is to be shared, the label may take center stage where it is translated from one mind to another. In an innovative study, Price and Djulbegovic (2017) analyzed forward- and back-translation of "evidence" from English to 9o other languages, finding in nearly $90 \%$ of cases that the analogous translation corresponded (either exclusively or interchangeably) to "proof." As Fox notes, such a label carries a connotation of definitive judgment rather than the defeasibility we should hope to assume of research findings, clinical observations, and scientific evidence in general. ${ }^{7}$ Given that "evidence" is unlikely to depart our vocabulary, emphasizing relationality and emergence as central to its conceptualization may help improve specificity in its communication. ${ }^{\text {iv }}$

Even within English we find semantic significance in our use of "evidence." In proposing my introductory definition, I avoid the metonymy of labelling the data themselves "evidence" out of caution. Though we often speak of a body of data constituting evidence in this manner, such figures of speech risk conflation of evidence with data when addressing characteristics of the former, a pervasive semantic hazard exposed by Braithwaite in his identification of "EBM's six dangerous words." ${ }^{, 2, \mathrm{v}}$ Indeed, current discussions of available evidence in medicine are typically fraught with ambiguity, such as where references to "more evidence" indicate neither a greater degree of evidential support nor more extensive data with clarity. Rather, we ought to say that data offer (or provide) evidence in relation to one or more hypotheses. A consequence of this distinction is that data may provide no evidence at all: in some trivial cases the relationality of evidence is clearly exploited, (e.g. irrelevant data) but the importance of relativity is reflected in the fact that relevant data may nonetheless be evidence-poor with respect to the hypotheses 
under consideration. ${ }^{5}$ Thus, to ask "what evidence is there?" is to call upon the available and relevant data and hypotheses within one's theoretical context to identify what support can be found. In agreement with Upshur (200o), ${ }^{8}$ evidence in this sense can be construed as an emergent property of the theory-hypotheses-data (or alternatively, background-propositionsobservations) system.

Whereas relationality is reflected in the proposed definition, relativity is only implied through invoking hypotheses in the plural; I think this is a reasonable price for its accessibility, and deferring discussion of evidential relativity may in fact contribute in this regard.

\section{Evidence in "Evidence-Based Medicine (EBM)"}

Gupta (2003) highlights the equation of evidence with "valid quantitative data" under the paradigm of EBM, and argues that "the gap between data and evidence is obscured by EBM's definition of 'evidence', which equates best research evidence with 'clinically relevant research."'13 Gupta suggests that evidence should instead be understood as "a status conferred upon a fact" where the latter is judged to "[increase] the likelihood of a given conclusion being true." ${ }^{\prime 3}$ Taking "fact" to imply accurate information, its influence on the validity of conclusions can be understood as necessitating its relevance to them.

At the outset of a Derridaean deconstruction of EBM, Devisch and Murray (2009) offer an alternative appraisal of the paradigm's "evidence," arguing that "The E of EBM therefore acts as an authoritative cipher, the synonym, for evidence in general: $E=$ truth $=$ reality." ${ }^{\prime 4}$ The authors' 
assertion that EBM is "committed to the belief that evidence speaks for itself" invites consideration of a disturbing potential consequence of EBM's standard curriculum: that the greater concept of evidence is ignored and a hierarchy of study designs deemed fundamental should be worrisome, as it virtually ensures the crude conclusion that evidence is simply data and results brought forth from studies. Furthermore, Worrall (2010) argues that such hierarchies give a false sense of specificity of the results to the hypotheses of interest. ${ }^{15}$ As Worrall notes, this issue of "external validity" intersects with that of generality, and the relationality of evidence obtained through medical research is not necessarily concordant with the "level of evidence" attributed to the underlying study design.

Fortunately, these consequences can be mitigated by giving "evidence" the time it deserves, and indeed I believe my suggestion demands very little. Rather than burden an already dense course of instruction with an excursion through philosophical theory, the introductory concept proposed here can be seamlessly included as a foundation for rational thinking in medicine and a stimulus for interested students to contemplate and read further on the subject.

\section{Conclusion}

Despite its titular inclusion in EBM curricula, the concept of evidence is often left undefined. Typical presentations of evidence hierarchies implying that "evidence" denotes data are potentially misleading, and fail to provide students the opportunity for critical reflection and self-directed exploration of theory. The evidence-as-property construction proposed here 
provides a clear and simple introductory definition to disambiguate "evidence" from a set of data and the inferential support they may offer.

\section{Footnotes}

i The use of "information" here appears ambiguous, as it might indicate either the subject of a support relation between data and hypothesis (i.e. the data) or the support per se.

${ }^{\text {ii }}$ Whereas Fox's construction is ostensibly intended as a pragmatic, simplifying one, I have not encountered formal constructions of evidence as support subject in the philosophy literature.

${ }^{\text {iii }}$ General distinctions between pure likelihood and Bayesian inferential paradigms notwithstanding.

iv More general terms might conceivably be of use, as in describing "support" provided by observations, such that ambiguous or self-referential use of "evidence" could be avoided.

$\mathrm{v}$ “There is no evidence to suggest...” 


\section{Acknowledgment}

I owe the inspiration for this essay to Dr. Daniël Lakens, (Eindhoven University of Technology, The Netherlands) whose writing first spurred me to consider the meaning of "evidence." 


\section{References}

1. Evidence-Based Medicine Working Group. Evidence-based medicine. A new approach to teaching the practice of medicine. JAMA 1992;268(17):2420-2425. doi: 10.1001/jama.1992.03490170092032

2. Sackett DL, Rosenberg WMC, Muir Gray JA, Haynes RB, Richardson WS. Evidence based medicine: What it is and what it isn't. BMJ 1996;312:71-2. doi: 10.1136/bmj.312.7023.71

3. Thistlethwaite J, Davies H, Dornan T, Greenhalgh T, Hammick M, Scalese R. What is evidence? Reflections on the AMEE symposium, Vienna, August 2011. Med Teach 2012;34:454-7. doi: 10.3109/0142159X.2012.671561

4. Kelly T. Evidence. The Stanford Encyclopedia of Philosophy (Winter 2016 Edition), Edward N. Zalta (ed.). https://plato.stanford.edu/archives/win2016/entries/evidence/

5. Morey RD, Romeijn J-W, Rouder JN. The philosophy of Bayes factors and the quantification of statistical evidence. J Math Psychol 2016;72:6-18. doi: 10.1016/j.jmp.2015.11.0o1

6. Macnaughton RJ. Evidence and clinical judgement. J Eval Clin Pract 1998;4(2):89-92. doi: 10.1111/j.1365-2753.1998.00002.x

7. Fox J. Arguing about the evidence: A logical approach. In: Dawid P, Twining WL, Vasilaki M, eds. Evidence, Inference, and Enquiry. Oxford: Oxford University Press; 2011.

8. Upshur REG. Seven characteristics of medical evidence. J Eval Clin Pract 2000;6(2):93-7. doi: 10.1046/j.1365-2753.2000.00244.x 
9. Upshur REG, VanDenKerhkhof EG, Goel V. Meaning and measurement: An inclusive model of evidence in health care. J Eval Clin Pract 2001;7(2):91-6. doi: 10.1046/j.13652753.2001.00279.x

10. Goodman SN, Royall R. Evidence and scientific research. Am J Public Health 1988;78:156874. PMCID: PMC1349737

11. Price AI, Djulbegovic B. What does evidence mean? Most languages translate "evidence" into "proof". J Eval Clin Pract 2017;23:971-73. doi: 10.1111/jep.12834

12. Braithwaite RS. EBM's six dangerous words. JAMA 2013;310(20):2149-50. doi: 10.1001/jama.2013.281996

13. Gupta M. A critical appraisal of evidence-based medicine: some ethical considerations. $J$ Eval Clin Pract 2003;9(2):111-21. doi: 10.1046/j.1365-2753.2003.00382.x

14. Devisch I, Murray SJ. 'We hold these truths to be self-evident': Deconstructing 'evidencebased' medical practice. J Eval Clin Pract 2009;15:950-4. doi: 10.1111/j.1365-2753.2009.01232.x 15. Worrall J. Evidence: Philosophy of science meets medicine. J Eval Clin Pract 2010;16:35662. doi: 10.1111/j.1365-2753.2010.01400.x 